\title{
Fluorescently labeled oligonucleotide extension: a rapid and quantitative protocol for primer extension
}

\author{
Richard A. Fekete, Mark J. Miller, and Dhruba K. Chattoraj
}

BioTechniques 35:90-98 (July 2003)

\begin{abstract}
Identification of nucleotides used for RNA chain initiation or for contacting DNA binding proteins is basic to our understanding of gene regulation. Normally, a radioactive primer is used to copy RNA or DNA. The polymerase extension stops at free ends of $m R N A$ (as in promoter mapping) or at the position of template cleavage or modification (as in footprinting). The locations of these positions are then analyzed by polyacrylamide gel electrophoresis. These analyses have been improved using fluorescently labeled primers and commonly available DNA sequencing machines. The protocol, which we call fluorescently labeled oligonucleotide extension (FLOE), eliminates the need for handling radioactivity and polyacrylamide. The DNA sequencer delivers data as a "trace" that is ready for quantification, which eliminates the need to trace gels separately. The data analysis is further improved by new software, Scanalyze, which we present here. We demonstrate that by using promoter mapping and footprinting, FLOE shortens experimental time, extends the stretch of analyzable sequence, and simplifies quantification compared to radioactive methods and is as sensitive in terms of detecting templates.
\end{abstract}

\section{INTRODUCTION}

The identification of nucleotide positions of RNA chain initiation or those contacted by DNA binding proteins, such as transcription factors, is of widespread use in molecular biology (1-3) and basic to our understanding of gene regulation. The nucleotide positions are routinely examined using primer extension (4), in which a radioactive primer is used to copy a RNA or DNA template. The synthesis continues until the polymerase reaches the end of the template (5) or encounters a roadblock. The latter can be from template modification [as in potassium permanganate footprinting (6)] or a protein bound to the template [as in toe-printing (7)]. The locations of these positions are then determined when the products are analyzed by PAGE (4). An imprint of the gel is then obtained by autoradiography or using a phosphorimager.

The commonly used primer extension procedure has been simplified us- ing fluorescently labeled oligonucleotides and a DNA sequencer (8-11). For ease of reference, we have termed the technique fluorescently labeled oligonucleotide extension (FLOE) and provide a comprehensive account of the technique and improvements in data analysis.

The use of a fluorescent label, as opposed to radioactive label, eliminates the need for hazardous radioactivity, allowing it to be performed on any laboratory bench. The use of the DNA sequencer also eliminates the need for handling large amounts of polyacrylamide. FLOE shortens experimental time by up to one day compared to conventional techniques because gels do not need to be poured, and no exposure to film or phosphorimaging plate is required. Time is also saved because the primers do not require repeated labeling, as is the case with radioactivity. Fluorescent tags have been used up to a year with no loss in signal intensity. Current techniques require that bands from an autoradiograph or phosphorimage be individually quantified in a labor-intensive process (12). Data from the DNA sequencer are delivered as a "trace" that can be used to directly quantitate the proportion of each species, providing additional timesavings during data analysis. The instrument also performs background measurements and their subtraction. The protocol is improved by using primers that are purified so that all contain labels. This ensures that all extension products are labeled, which allows us to detect template concentrations as low as is achieved using radioactive protocols. FLOE also increases the range of analyzable sequence using a single primer and a single loading. In addition to technical and safety improvements, the wide availability of DNA sequencers makes FLOE a generally applicable protocol.

FLOE can be applied to map promoters and to locate DNA modifications or cleavage sites produced by any of the 
commonly used agents, such as DNase I, hydroxyl radical, dimethyl sulfate (DMS), and potassium permanganate. Secondary structure analysis of RNA, which uses enzymes to cleave specific structures, can also benefit from this simplified technique. The applicability of FLOE is documented here using wellcharacterized sites of transcription and replication in Escherichia coli.
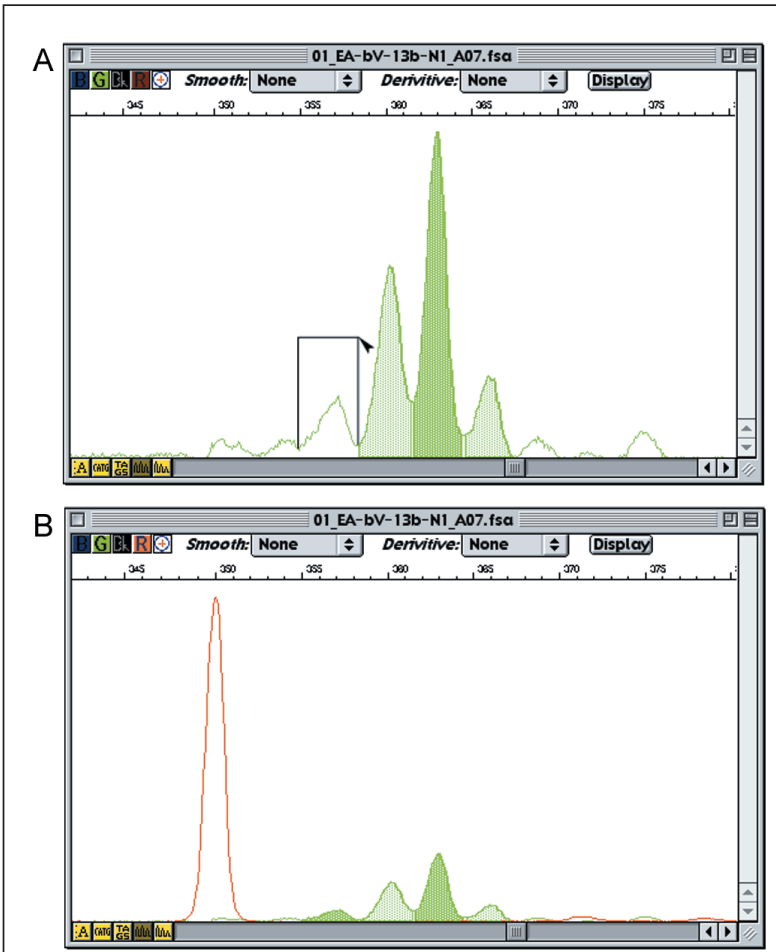

C

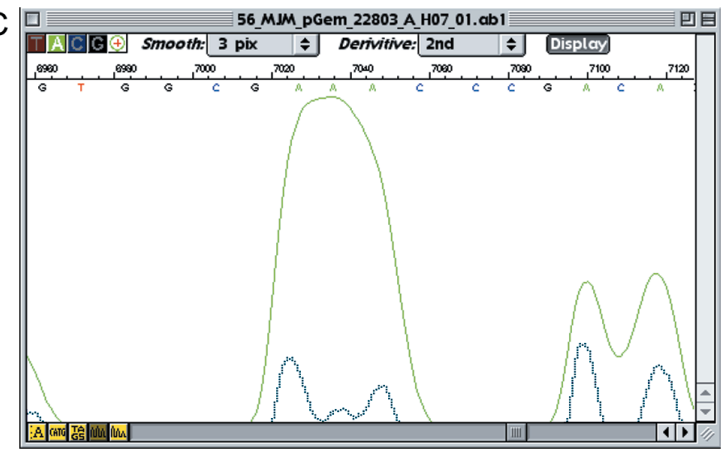

Figure 1. Key features of the data analysis software, Scanalyze. (A) Neighboring peaks can be shaded in light and dark green for clarity. In addition, an authentic peak, if missed by the Applied Biosystems' program, can be included in the analysis by using the mouse cursor to define the peak limits (boxed region). The program checks to ensure that the new peak does not overlap any other and asks the user if the peak limits are acceptable (data not shown). (B) Selective display of traces. Red trace, not displayed in panel A, is added in panel B. In addition, the auto-zoom mode allows the window scale to be set so the maximum data point in the window is close to the top of the display. (C) Peak resolution using second derivatives. The negative core of the second derivative for one of the bases (A) from a sequencing gel is displayed as a blue dashed line. The sequence given by the automatic base caller in GeneScan is shown above the trace. Note that the three A's in the center of the figure (sequence,...CGAAACCC...) appear as a single, smeared peak in the original, green solid data line, but are readily distinguishable when the negative cores are displayed.

\section{MATERIALS} AND METHODS

\section{Preparation of} Nucleic Acids

RNA was synthesized in vitro using E. coli RNA polymerase (USB, Cleveland, OH, USA), following the supplier's protocol. When re- quired, cAMP receptor protein (CRP) was used at $5 \mathrm{nM}$. RNA was isolated from cells grown in LB medium to mid-log phase using an RNeasy ${ }^{\circledR}$ kit (Qiagen, Valencia, CA, USA). DNase I footprinting was performed, as recommended in the Promega technical bulletin no. 137 (http://www.promega. com/tbs/tb137/tb137.html). The buffer for footprinting was composed of 20 $\mathrm{mM}$ Tris, $\mathrm{pH}$ 7.5, $100 \mathrm{mM}$ potassium glutamate, $10 \mathrm{mM}$ magnesium acetate, $1 \mathrm{mM}$ calcium chloride, $1 \mathrm{mM}$ DTT, $100 \mu \mathrm{M}$ ATP, $0.1 \%$ (v/v) Igepal ${ }^{\circledR}$ detergent (Sigma, St. Louis, MO, USA), and $10 \%(\mathrm{v} / \mathrm{v})$ glycerol. Digested DNA was purified using phenol:chloroform extraction and ethanol precipitation, and then it was used as template for primer extension in a thermal cycler.

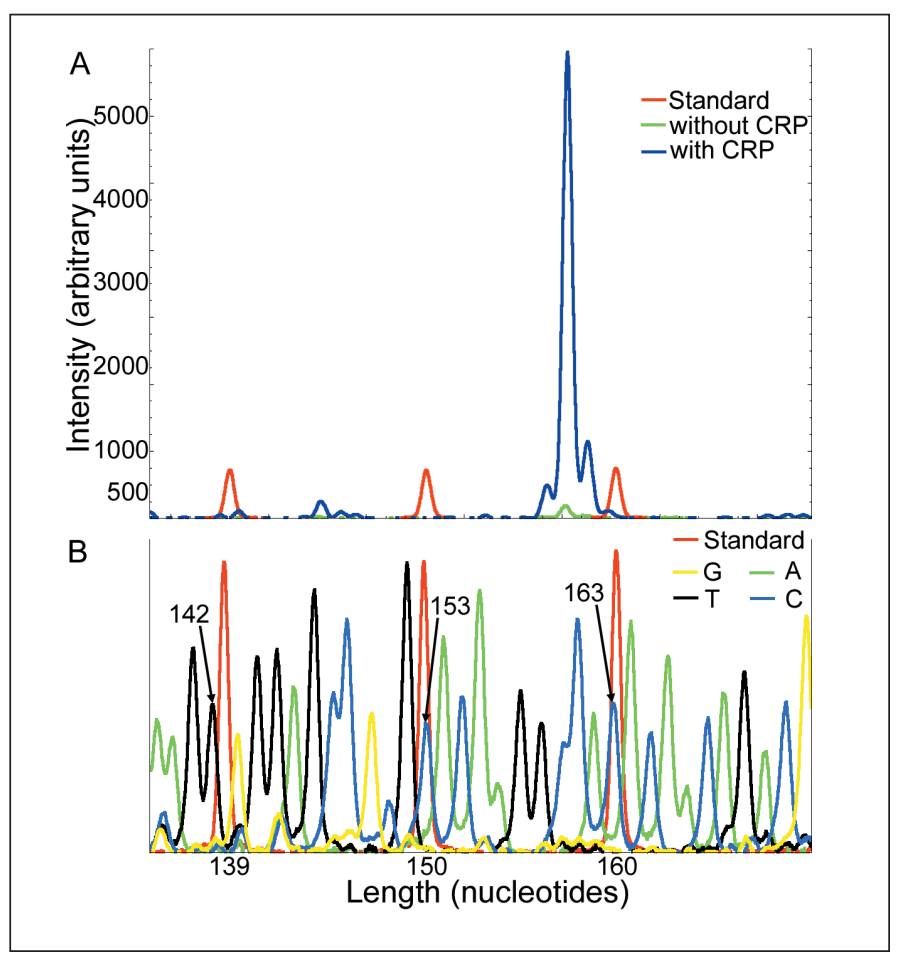

Figure 2. Promoter mapping using primer extension. FLOE was used to reverse transcribe RNA synthesized in vitro from the lac promoter, and the products were analyzed using a DNA sequencer. (A) Traces from the DNA sequencer representing products from reverse transcription reactions using RNA synthesized in the presence (blue trace) and in the absence (green trace) of cAMP receptor protein (CRP). The samples were run separately, and then traces were overlaid for ease of comparison. The red trace represents the Applied Biosystems' size standards that were included in each sample, and their sizes are marked on the $\mathrm{x}$-axis. The standard also helped to align the traces. The y-axis shows band intensities in arbitrary units. (B) The determination of apparent migration of the size standards. A dideoxy sequence ladder is generated, using the same template and primer that were used for promoter mapping, and mixed with the size standards. The arrows define the nucleotide lengths of the sequencing products that co-migrate with the size standards. The size standards migrate as if they are three nucleotides longer than their actual nucleotide length. 


\section{Primer Extension}

In all the FLOE experiments reported here, the primers had a fluorescent dye (6-Fam) covalently linked to the $5^{\prime}$ end (Sigma-Genosys, The Woodlands, TX, USA). We have also tried the dye, Hex, with success. The emission spectra of both of these dyes are easily separable from that of the Applied Biosystems' size standards (Foster City, CA, USA) described below. For RNA templates, primer extension reactions used the Reverse Transcription system (Promega, Madison, WI, USA). For PCR, ThermalAce ${ }^{\mathrm{TM}}$ DNA polymerase (Invitrogen, Carlsbad, CA, USA) was used in $20 \mu \mathrm{L}$ containing $100 \mathrm{pmol}$ primer. The same primer was used in sequencing reactions performed using the fmol DNA Cycle Sequencing kit (Promega).

When comparing radioactive primer extension to FLOE, primers of identical length and sequence were labeled with $\left[\gamma^{32} \mathrm{P}\right]$ ATP or 6 -Fam. Radioactive endlabeling was performed using T4 Polynucleotide Kinase (New England Biolabs, Beverly, MA, USA) with radionucleotides of the highest specific activity available $(>5000 \mathrm{Ci} / \mathrm{mmol})$ (Amersham Biosciences, Piscataway, NJ, USA) and following the protocol given in the Reverse Transcription system Promega technical bulletin no. 113 (http://www.promega.com/tbs/tb113/ tb113.html). The specific activity of the primer $\left(1.725 \times 10^{6} \mathrm{cpm} / \mathrm{pmol}\right)$ was determined by trichloracetic acid (TCA) precipitation as previously described (13). RNA was isolated from cells containing pLacIa and pUC19 after a 3-h induction with $0.5 \mathrm{mM}$ IPTG. During extension reactions, to map the lac promoter on pUC19, 7 and $0.7 \mu \mathrm{g}$ total RNA were used with 2.5 pmol radioactive or fluorescently labeled primer. Radioactive primer extension reactions were concentrated, and the entire sample run on a $6 \%$ denaturing polyacrylamide gel. The gel was dried and then exposed to a BAS phosphorimager plate (Fuji, Tokyo, Japan).

\section{DNA Electrophoresis of Fluorescent Products}

All reaction products were purified using Performa ${ }^{\mathbb{R}}$ DTR Gel Filtration cartridges (Edge BioSystems, Gaithersburg, MD, USA) and kept frozen at $-20^{\circ} \mathrm{C}$ until use. Samples were separated on $50-\mathrm{cm}$ capillary columns in the POP4 acrylamide polymer (Applied Biosystems) on an ABI PRISM ${ }^{\circledR} 3100$ Genetic Analyzer (Applied Biosystems) capillary electrophoresis instrument using techniques and parameters suggested by the manufacturer. The ROX-500 Size Standards (Applied Biosystems) were included in each run and used to determine fragment lengths.

\section{Data Analysis}

GeneScan ${ }^{\circledR}$ version 3.1.2 (Applied Biosytems) was used to initially screen the data and identify major peaks. However, to precisely determine fragment lengths and amounts, Scanalyze version 0.4 [a sequence and microsatellite analysis program currently being developed by one of us (M.J.M., mark@mail.nih.gov)] was used. This program allows for the analysis of data files produced by the Applied Biosystems' genetic analysis instruments. It works on either Sequencing or GeneScan files and is written to supplement the Applied Biosystems data analysis programs by allowing the investigator to edit peaks that are recognized by the standard Applied Biosystems' programs (Figure 1A).

Scanalyze contains several features that help view and interpret data. A zooming tool allows one to quickly magnify features of interest. An "auto-zoom" mode automatically scales the display to include the maximum point in the current window (Figure 1B). The ability to selectively display traces also simplifies analysis (e.g., the addition of the red trace in Figure 1B to the green trace of Figure 1A). One can also overlay the first or second derivatives of the trace data using Savitzky-Golay smoothing filters (14). In particular, the second derivative display can be used as a powerful contrast-enhancing technique that allows the separation of shoulders on adjacent peaks (Figure 1C).

Scanalyze allows the chromatograms, or analysis results, to be exported in a form readable by most generalpurpose graphics programs or spreadsheets. In the present examples, experimental data was exported from 
Scanalyze into KaleidaGraph ${ }^{\circledR}$ (version 3.0; Synergy Software, Reading, PA, USA), allowing difference curves to be plotted and different runs to be overlayed using the Applied Biosystems' size standards as guides (Figures 2-5).

\section{RESULTS AND DISCUSSION}

To test whether FLOE can map the start site of a transcript accurately, we mapped the well-studied lac promoter by primer extension. The template was the frequently used cloning vector pUC19 that contains the wild-type lac promoter (New England Biolabs). RNA was synthesized in vitro with and without CRP [CRP is required for the activation of the lac promoter (15)] The RNA samples were then used in extension reactions using reverse transcriptase and a primer with a fluorescent dye (6-Fam) covalently linked to its $5^{\prime}$ end. Reverse transcription products were purified, combined with the size standards, and then run on the ABI PRISM 3100 Genetic Analyzer.

The data are obtained as a trace of fluorescent intensities that correspond to DNA fragments of different lengths and amounts. The trace was initially analyzed using the GeneScan software. Subsequently, Scanalyze was used to precisely determine the lengths and amounts of DNA, corresponding to individual bell-shaped curves of the trace (see Materials and Methods). To determine the DNA fragment lengths, the software creates a standard curve using the length of the Applied Biosystems' DNA size standards versus their respective mobility. The size of experimental DNA is then determined by interpolation. The amount of experimental DNA was simply the area under the curves in arbitrary units. Scanalyze also allowed for the printing of selected regions of the trace and export of the data to a spreadsheet program for further manipulation of the data. Figure $2 \mathrm{~A}$ is a sample of the output from the DNA sequencer after export into KaleidaGraph (a graphing program). A strong peak can be seen at a position of 157.3 nucleotides when the RNA was synthesized in the presence of CRP (blue trace). The peak intensity was considerably lower in the absence of CRP (green trace), which confirmed the CRP dependence of lac promoter activity (15). The two traces were overlaid using the size standards (red trace) as a guide.

To identify the product size correctly, a standard dideoxy sequencing reaction was performed, utilizing the same primer used during reverse transcription. Products of the sequencing reaction were

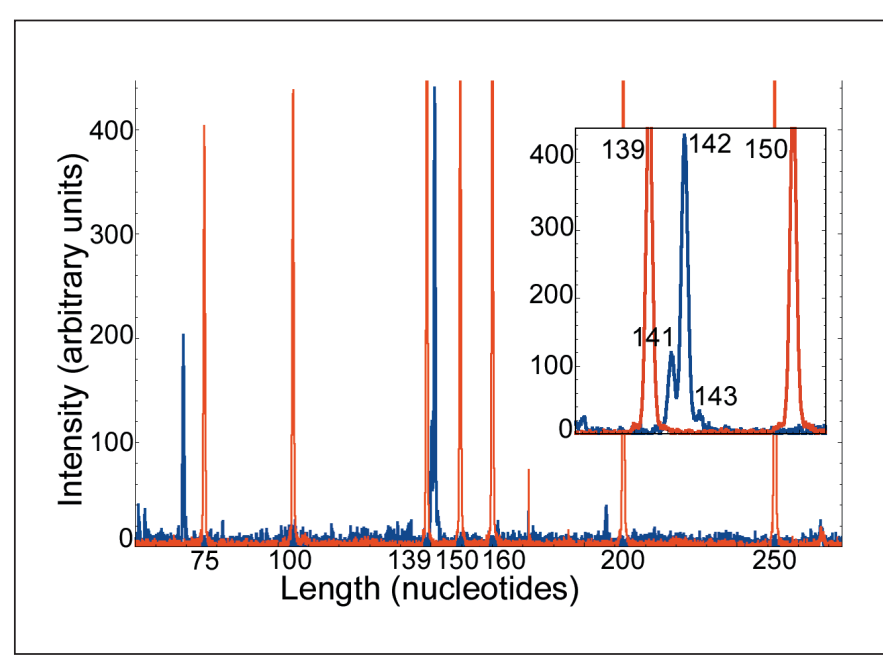

Figure 3. Promoter mapping using total cellular RNA. As in Figure 2, except that total cellular RNA was used to measure the activity of a weak promoter (repA) in vivo. Shown are the traces for the repA promoter (blue) and the size standards (red). Inset: a magnification of the trace from nucleotide positions 135-150. purified, combined with the GeneScan size standards, and run on the DNA sequencer. The fluorescently labeled Applied Biosystems' size standards ran as if they were, on an average, three nucleotides longer than the dideoxy sequencing products (Figure 2B). When corrected for this difference, transcription was found to begin primarily with the sequence GAATTG, which is identical to the preferred sequence found in vitro and in vivo $(16,17)$.

We next applied FLOE to total RNA isolated from cells containing a P1 plasmid promoter (repAP) fused to $l a c Z$ (18). This promoter provides approximately an order of magnitude less activity than the unrepressed lac promoter (data not shown). RNA was isolated from cells in mid-log phase, and extension reactions were performed as described earlier. Data from the DNA sequencer are shown in Figure 3, and a magnification of the peak seen at nucleotide position 142 is inset (not sizecorrected). Again, a dideoxy sequencing reaction was performed and run on the DNA sequencer (data not shown).

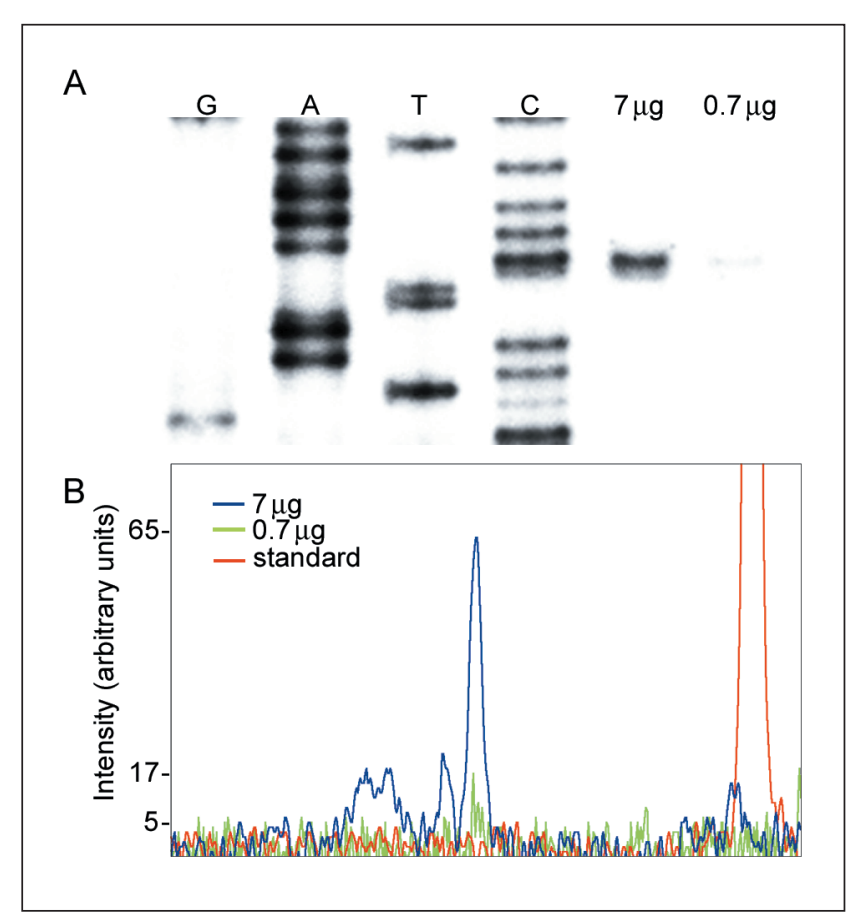

Figure 4. Detection limits of primer extension using radioactive versus fluorescent primers and $l a c$ mRNA. (A) PAGE of primer extension reactions using 7 and $0.7 \mu \mathrm{g}$ total cellular RNA with a radioactive primer and a 16-h exposure. A sequencing reaction is run alongside as a standard. (B) Identical to panel A, except the primer has a fluorescent label. Reactions using 7 (blue trace) and $0.7 \mu \mathrm{g}$ (green trace) of RNA are shown. One of the size standards is also shown (red trace). 
When the mobility difference, in comparison to the Applied Biosystems' size standards was accounted, the transcriptional start site matched that previously published (19). The galP1 and P2 promoters were also correctly mapped using FLOE from RNA isolated in vivo (data not shown) (20).

To confirm the accuracy of FLOE for determining product length, pUC19 was digested with HindIII and used as a template for primer extension using the same fluorescently labeled oligonu- cleotide as above. A peak matching the correctly sized fragment (99 nucleotides) was obtained after the data was corrected using a dideoxy sequencing standard (data not shown).

To demonstrate the reproducibility of FLOE, lac promoter mapping was repeated four times in independent extension reactions, and the mean product length was found to be $157.4 \pm$ 0.06 nucleotides. Mapping of the repA promoter was also repeated in six independent extension reactions, and the mean was $141.8 \pm 0.05$ nucleotides.

FLOE was compared to a radioactive method to determine the ability of each to detect low levels of mRNA. Total cellular RNA was isolated from cells expressing lac from pUC19. When a radioactive primer was used to map the lac promoter, the reactions were concentrated, and the entire samples were run on a denaturing polyacrylamide gel (Figure 4A). After exposure for $16 \mathrm{~h}$, products using $7 \mu \mathrm{g}$ RNA were easily detected. However,

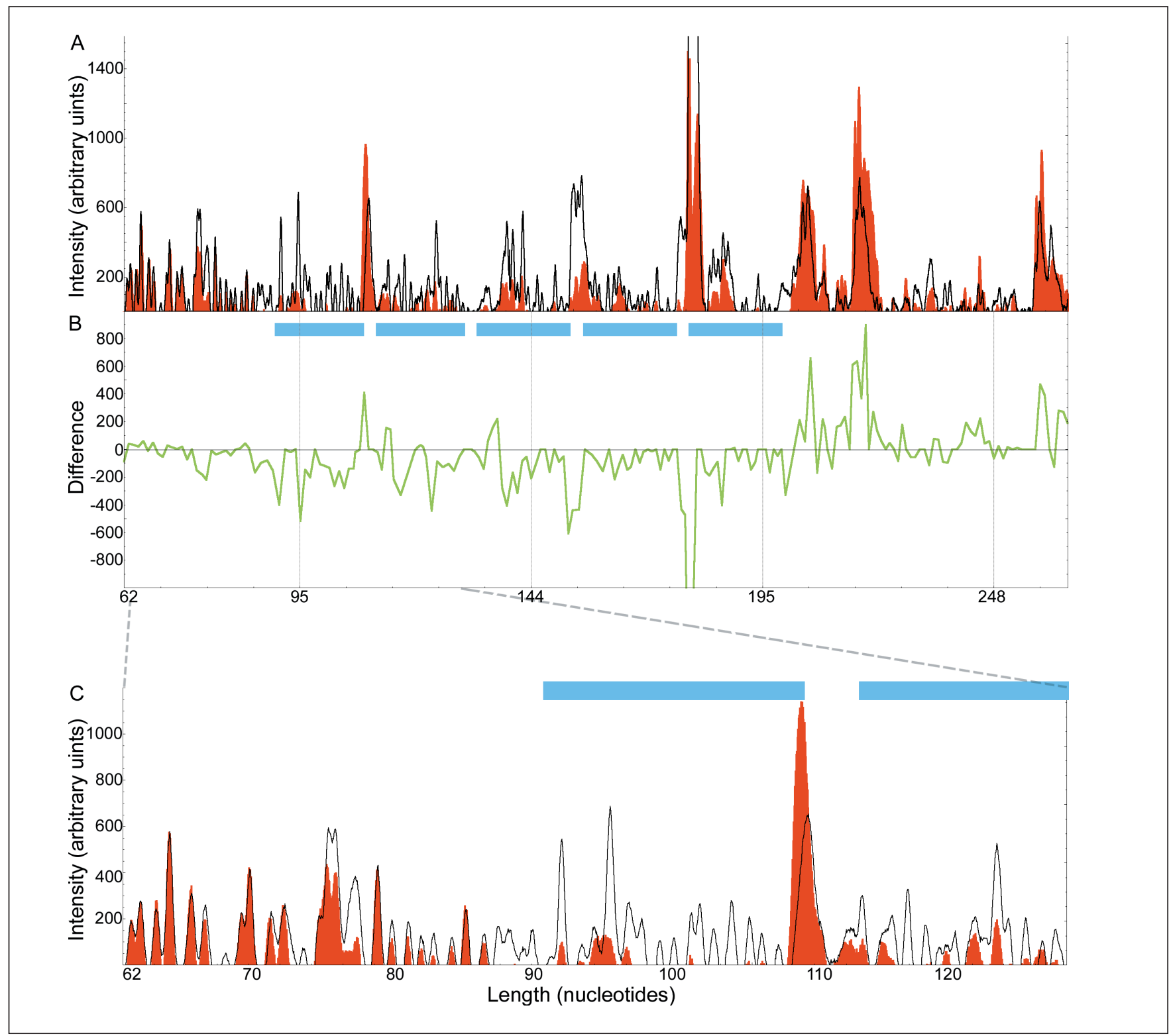

Figure 5. In vitro DNase I footprinting of RepA on its binding sites at the P1 plasmid origin. (A) The plasmid was treated with DNase I in the presence (red trace) and absence (black trace) of RepA and subjected to FLOE. The RepA binding sites are shown with solid bars. (B) A difference curve of the intensities in panel A, calculated by quantifying the height of each peak and subtracting the values obtained without RepA from the values with RepA. (C) A magnified view of panel A, spanning the region 62-129 nucleotides. Note the hypersensitive site at position 109 that was also seen using PAGE (21). 
detection became difficult when 10 times less template was used, unless the exposure time was extended. Upon quantification, this band was approximately 3 -fold above background (129 vs. 44 arbitrary units). When an identical primer but labeled with 6-Fam was used, reactions with $7 \mu \mathrm{g}$ RNA (Figure $4 \mathrm{~B}$, blue trace) were easily detected. Products were also detectable using 0.7 $\mu \mathrm{g}$ RNA (green trace). This signal was 3 -fold above background when peak heights were measured (17 vs. 6), and also when peak areas were quantitated (76 vs. 26). The area of the peak corresponding to the $0.7-\mu \mathrm{g}$ reaction was approximately 10 -fold less than that of the $7-\mu \mathrm{g}$ reaction (860 vs. 76 ). This shows that FLOE can detect low levels of template as quantitatively as the radioactive method, without requiring lengthy exposure.

We next applied FLOE to monitor binding of a P1 plasmid protein, RepA, to the plasmid origin in vitro. DNase I footprinting was performed in the presence and absence of RepA (Figure 5A). When the apparent mobility of the Applied Bioystems' size standards was corrected using a dideoxy sequencing control (data not shown), the FLOE-derived footprint mirrored that observed previously using PAGE (21). FLOE allowed for the analysis of up to $300 \mathrm{nu}-$ cleotides from the primer. Footprinting of the $\mathrm{P} 1$ origin was also done in vivo using DMS, and the cleavage positions were resolvable up to 400 nucleotides from the primer (data not shown). This is a considerable improvement over the resolvable region obtained using radioactive primers and PAGE (21). Similar improvement has been realized with fluorescent versus radioactive sequencing (22-24). Figure 5B shows a difference curve that was obtained by subtracting the peak intensity values of cleavage without RepA from the values with RepA using KaleidaGraph. Values close to zero indicate equal cleavage in the presence and absence of RepA (no protection). Values above and below zero represent hypersensitive and protected regions, respectively. The predominance of the curve below zero in the area of the RepA binding sites shows that DNase I cleavage in this area is considerably reduced in the presence of RepA. The protection by
RepA is better illustrated in a magnification of a small section of the trace (Figure 5C).

\section{ACKNOWLEDGMENTS}

We thank Dale Lewis (LMB, NCI) for help with the in vitro transcription and PAGE, members of the laboratory, and C.A. Hamilton for helpful suggestions. CRP was a gift from M. Soukhodolets and M.F. Liu (LMB, NCI).

\section{REFERENCES}

1.McKnight, S.L. and R. Kingsbury. 1982. Transcription control signals of a eukaryotic protein-encoding gene. Science 217:316-324

2.Chai, X., W. Chen, and J.L. Napoli. 2001. Structure, promoter and chromosomal localization of $r d h 6$. Gene 274:27-33.

3.Galas, D.J. 2001. The invention of footprinting. Trends in Biochem. Science 26:690-693.

4.Sambrook, J., E.F. Fritsch, and T. Maniatis. 1989. Molecular cloning: a laboratory manual, 2nd ed. CSH Laboratory Press, Cold Spring Harbor, NY.

5.Boorstein, W.R. and E.A. Craig. 1989 Primer extension analysis of RNA. Methods Enzymol. 180:347-369.

6.Sasse-Dwight, S. and J.D. Gralla. 1991 Footprinting protein-DNA complexes in vivo. Methods Enzymol. 208:146-168.

7.Pestova, T.V., C.U. Hellen, and I.N. Shatsky. 1996. Canonical eukaryotic initiation factors determine initiation of translation by internal ribosomal entry. Mol. Cell Biol. 16:6859 6869.

8.Sandaltzopoulos, R., W. Ansorge, P.B. Becker, and H. Voss. 1994. Nonradioactive, solid-phase DNase I footprints analyzed on an A.L.F. DNA sequencer. BioTechniques 17:474-478.

9.Altermann, E., J.R. Klein, and B. Henrich. 1999. Synthesis and automated detection of fluorescently labeled primer extension products. BioTechniques 26:96-101.

10.Yindeeyoungyeon, W. and M.A. Schell. 2000. Footprinting with an automated capillary DNA sequencer. BioTechniques 29:10341041.

11.Yam, J.W., K.W. Chan, and W.L. Hsiao. 2001. Incorporating the ABI GeneScan analysis to a RACE-based technique for mapping multiple transcription initiation sites. Mol. Biotechnol. 17:129-134.

12.Storek, M.J., A. Ernst, and G.L. Verdine. 2002. High-resolution footprinting of sequence-specific protein-DNA contacts. Nat. Biotechnol. 20:183-186.

13.Mukhopadhyay, G., J.A. Dibbens, and D.K. Chattoraj. 1995. Protein-protein interactions of DNA-binding proteins: studies on the replication initiator protein, RepA, of plasmid P1. Methods in Molecular Genetics 6:400-420. Academic Press, NY.

14.Savitzky, A. and M.J.E. Golay. 1964.
Smoothing and differentiation of data by simplified least squares procedures. Anal. Chemist. 36:1627-1639.

15.Joung, J.K., L.U. Le, and A. Hochschild. 1993. Synergistic activation of transcription by Escherichia coli cAMP receptor protein. Proc. Natl. Acad. Sci. USA 90:3083-3087.

16.Carpousis, A.J., J.E. Stefano, and J.D. Gralla. 1982. 5' nucleotide heterogeneity and altered initiation of transcription at mutant lac promoters. J. Mol. Biol. 157:619-633.

17.Xiong, X., N. de la Cruz, and W.S. Reznikoff. 1982. Downstream deletion analysis of the lac promoter. J. Bacteriol. 173:45704577.

18.Papp, P.P., G. Mukhopadhyay, and D.K. Chattoraj. 1994. Negative control of plasmid DNA replication by iterons. Correlation with initiator binding affinity. J. Biol. Chem. 269:23563-23568

19.Chattoraj, D.K., K.M. Snyder, and A.L. Abeles. 1985. P1 plasmid replication: multiple functions of RepA protein at the origin. Proc. Natl. Acad. Sci. USA 82:2588-2592.

20.Lewis, D.E.A., M. Geanacopoulos, and S. Adhya. 1999. Role of HU and DNA supercooiling in transcription repression: specialized nucleoprotein repression complex at gal promoters in Escherichia coli. Mol. Microbiol. 31:451-461.

21.Mukhopadhyay, G., K.M. Carr, J.M. Kaguni, and D.K. Chattoraj. 1993. Open-complex formation by the host initiator, DnaA, at the origin of P1 plasmid replication. EMBO J. 12:4547-4554.

22.Griffin, H.G. and A.M. Griffin. 1993. DNA sequencing. Recent innovations and future trends. Appl. Biochem. Biotechnol. 38:147159.

23.Ansorge, W., B.S. Sproat, J. Stegemann, and C. Schwager. 1986. A non-radioactive automated method for DNA sequence determination. J. Biochem. Biophys. Methods 13:315-323.

24.Ansorge, W., A. Rosenthal, B. Sproat, C. Schwager, J. Stegemann, and H. Voss. 1988. Non-radioactive automated sequencing of oligonucleotides by chemical degradation. Nucleic. Acids Res. 16:2203-2206.

Received 1 April 2003; accepted 22 April 2003.

Address correspondence to:

Dr. Richard A. Fekete

Laboratory of Biochemistry

National Cancer Institute

National Institutes of Health

Bethesda, MD 20892-4255, USA

e-mail:feketer@mail.nih.gov 